DOI 10.37882/2223-2974.2020.11.19

\title{
НАПРАВЛЕНИЯ ПОВЫШЕНИЯ РЕНТАБЕЛЬНОСТИ РЕАЛИЗАЦИИ РЕСУРСОВ ПРЕДПРИНИМАТЕЛЬСКИХ СТРУКТУР
}

\section{DIRECTIONS OF INCREASING THE PROFITABILITY OF THE SALES \\ OF ENTREPRENEURIAL STRUCTURES RESOURCES}

\section{E. Ksenofontova}

A. Efimov

Summary: Various economic indicators can be used to determine the performance of a company. The authors note that one of the most important indicators of increasing the competitiveness of entrepreneurial structures is the profitability of the sale of resources. The article discusses ways to increase the profitability of the sale of resources of entrepreneurial structures by: changing the difference in the value between revenue and the cost of production, planning the volume and price of products by calculating the break-even point, changing non-production, production and internal factors. Increasing the efficiency of the company is impossible without increasing the profitability of personnel, and in particular, increasing the profitability of each employee, which is used to calculate the required number of personnel, their workload, identify human potential, and search for possible ways to increase personnel productivity. The article pays special attention to methods and measures to increase the profitability of entrepreneurial structures, such as improving product quality, improving product properties, reducing costs, reducing labor costs, and developing a marketing policy. Much attention is paid to the analysis of the profitability of assets, such as the profitability of fixed assets. The authors note that this indicator shows the effectiveness of the policy in the field of assets of entrepreneurial structures, the degree of efficiency and rationality of the use of certain fixed assets, and analysis of the profitability from their use. Modern entrepreneurial structures should pay special and reverent attention to increasing the profitability of the sale of resources, promptly and flexibly influence negative changes, implement and carry out measures to increase the profitability of assets, which will be expressed in the effective management of the company itself.

Keywords: profitability, business structures, net profit, fixed assets.

\author{
Ксенофонтова Екатерина Андреевна \\ соискатель, Санкт-Петербургский государственный \\ экономический университет \\ tyuksenofontova@mail.ru \\ Ефимов Алексей Александрович \\ аспирант, Санкт-Петербургский государственный \\ экономический университет
}

Аннотация: Для определения эффективности деятельности компании можно применять разные экономические показатели. Авторы отмечают, что одним из важнейших показателей повышения конкурентоспособности предпринимательских структур является рентабельность реализации ресурсов. В статье рассматриваются пути повышения рентабельности реализации ресурсов предпринимательских структур за счет: изменения разницы величины между выручкой и себестоимостью продукции, планирования объемов и цены продукции за счет расчета точки безубыточности, изменения непроизводственных, производственных и внутренних факторов. Повышение эффективности деятельности компании невозможно без увеличения рентабельности персонала, а в частности повышения рентабельности каждого сотрудника, которая применятся расчета необходимого количества персонала, их загрузки, выявления кадрового потенциала, поиска возможных путей увеличения продуктивности персонала. В статье уделено особое внимание на методы и мероприятия для повышения рентабельности предпринимательских структур, такие как повышение качества продукта, улучшение свойств продукта, снижение себестоимости, снижение расходов на оплату труда, разработка маркетинговой политики. Широкое внимание уделено анализу рентабельности активов, таких как рентабельность основных производственных фондов. Авторы отмечают, что этот показатель показывает результативность политики в сфере активов предпринимательских структур, степень эффективности и рациональности использования отдельных основных средств, проведения анализа доходности от их использования. Современные предпринимательские структуры должны уделять особое и трепетное внимание повышению рентабельности реализации ресурсов, оперативно и гибко влиять на негативные изменения, внедрять и проводить мероприятия по увеличению рентабельности активов, которое выразится в эффективное управление деятельности самой компании.

Ключевые слова: рентабельность, предпринимательские структуры, чистая прибыль, основные фонды.

Существуют отрасли хозяйства, где высокий рейтинг рентабельности отраслей. Высокая рентабельность обоснована высокой долей прибыли в доходах предпринимательской структуры, несмотря на высокие налоговые ставки, в табачной, алкогольной и других отраслях. В данной ситуации высокая рентабельность бизнеса обуславливается разницей себестоимости продукции и выручки. Высокий и достаточно стабильный спрос на продукцию позволяет компаниям закладывать большую долю прибыли. Государственный аппарат пытается изы- 
скать дополнительные доходы в бюджет страны и увеличивает налоговую ставку, что в свою очередь увеличивает цену. Несмотря на это спрос остается достаточно высоким, благодаря чему подобные отрасли остаются в рейтинге высокорентабельных отраслей.

Стоит отметить, что показатели высокой рентабельности обуславливается не размером дохода предпринимательской структуры, а скорее величиной разницы между выручкой и себестоимостью продукции. При изменении одного из показателей, ситуация в предпринимательской структуре может измениться и может возникнуть острая необходимость изменений. Можно отметить, что обусловленная разница между величинами достигается эффектом масштаба. Как правило, отрасли ориентируются на крупные партии проданных изделий. Отрасли, оказывающие услуги, также могут входить в список высокорентабельных. Как правило, высокая рентабельность отраслей, связанных с оказанием услуг, обуславливается относительно низкой себестоимостью. Услуги с высокой рентабельностью задействуют, как правило, информационные и трудовые ресурсы. Соответственно, затраты на предоставление услуги получаются не большого объёма и себестоимость предоставляемой услуги также оказывается не высокой, что дает возможность отраслям покрывать риски и входить в список высокорентабельных.

Раскрывая тему рентабельности финансово-хозяйственной деятельности предпринимательской структуры, прочих показателей эффективности ее деятельности, невозможно не отметить такой показатель, как точка безубыточности. Данный показатель определяет значение прибыльности от реализации произведенных товаров или услуг и отражает уровень цены, себестоимости продукта и объема продаж, при которых издержки предприятия будут равны прибыли от реализации, то есть прибыль будет равна нулю. Благодаря этому показателю, руководство предпринимательской структуры может взвешенно разработать стратегию ценообразования, значения скидок, и в целом, безошибочно определить наиболее выгодную цену на товар. Этот показатель зачастую используется для обоснования инвестиционных проектов, за счет этого показателя можно продемонстрировать точные объем продаж организации при определенном уровне затрат для получения нулевой прибыли от реализации проекта.

Подробный расчет показателей рентабельности позволяет оценить эффективность деятельности конкретной предпринимательской структуры. Но грамотно выстроенные и детально проработанный бизнес-план не дает гарантии на осуществление деятельности организаций без каких-либо проблем и условий. Для каждого предпринимателя важно иметь исчерпывающие знания о деятельности на выбранном рынке и оценивать всевоз- можные риски, которые могут повлиять на рентабельность проекта. Для этого стоит отметить, что существует совокупность факторов, которые могут положительно или отрицательно влиять на рентабельность. Эти факторы достаточно подробно отражены на рисунке 1.

Одним из основных и важнейших ресурсов в любой предпринимательской структуре является эффективный трудовой ресурс. К нему относят рабочих, специалистов, и других работников, которые входят в штат персонала организации. От компетентности и квалификации персонала во многом зависит успех предприятия, поэтому, безусловно, трудовым ресурсам нужно уделять большое внимание. Но стоит отметить, что помимо учета личных и профессиональных навыков работников, работодателю важно понимать, насколько ему выгодно работать с определенным штатом. Определить это позволяет такой показатель, как рентабельность персонала. Он дает возможность оценить эффективность вложения денежных средств в трудовой потенциал.

Рентабельность персонала определяется как отношение чистой прибыли к списочному числу рабочих, умноженное на 100 процентов. Результат этой формулы отражает выработку в среднем по одному сотруднику предпринимательской структуры. Также, стоит отметить, что показатели по конкретным работникам могут отличаться, для этого стоит вычислить рентабельность конкретного сотрудника, которая рассчитывается как отношение расходов на сотрудника, которые состоят из заработной платы, социальных выплат, издержек по всем пунктам и т. д., чистой прибыли, который принес этот сотрудник. Таким образом, руководитель может иметь полную картину по каждому работнику в своей организации и с учетом результатов вносить корректировки и поправки в рабочий процесс.

Безусловно, в каждой организации, важно не только вычислять, но и анализировать данный показатель. Анализ рентабельности персонала проводится с целью определения оптимальной численности работников, выявления кадрового потенциала, а также для поиска возможностей увеличения производительности труда и эффективности деятельности предпринимательской структуры [2].

Если, в ходе анализа, были обнаружены определенные проблемы, которые снижают эффективность деятельности организации, то стоит обратить внимания на способы повышения рентабельности персонала:

- изменение оплаты труда;

- совершенствование оборудования и рабочего места;

- повышение квалификации сотрудников;

- изменения графика работы;

- мотивационные корпоративные мероприятия, 
для поднятия морального духа штата.

В целом стоит отметить, что детально проработанная кадровая политика в предпринимательской структуре, сможет обеспечить эффективность работы персонала. Размер такого показателя, как рентабельность персонала напрямую связана с показателями развития организационного потенциала, который, в свою очередь, включает в себя уровень развития производственной техники на предприятии, материальное поощрение работников и социальные и корпоративные мероприятия, которые смогут показать работникам заинтересованность работодателя в своем персонале [3].

Показатель рентабельности очень важен при проведения первичного анализа деятельности предприятия и отрасли в целом. Он отражает эффективность работы предприятия, указывает на определенные недостатки в процессе производства продукции, управления ресурсами организации.

Рентабельность финансово-хозяйственной деятельности предпринимательской структуры является обобщающим показателем его экономической эффективности. Существуют различные пути улучшения рентабельности, исходя из имеющихся у организации ресурсов. Можно упомянуть о «money making»- ежедневных действиях, увеличивающих прибыль. Зачастую это незаметные и рутинные действия, которые в совокупности дают большой эффект и приводят к увеличению прибыли в компании.

Наиболее важные методы увеличения дохода предприятия, на наш взгляд, приведены на рисунке 2.

Важную роль в анализе деятельности компании играет анализ ее активов. Для первичного анализа активов используют показатель рентабельности активов, показывающий результативность политики в сфере активов предпринимательской структуры. Благодаря данному показателю, возможно выявить актив, который необходимо продать, сдать в аренду или вовлечь в производство. Показатель может выявить снижение оборачиваемости активов, посредством падения рентабельности. Изменение стоимости активов также может отразиться на их рентабельности. Уменьшение рентабельности активов влечет за собой потерю денежных средств. Для ведения успешной деятельности и отсутствия денежных потерь компании необходимо контролировать данный показатель. При его снижении необходимо провести анализ активов и исправить сложившуюся ситуацию. Неиспользуемый актив негативно сказывается на рентабельности и прибыли, так как компания не получает доход. В данной ситуации компании предпочитаю сдать

\section{Непроизводственные}

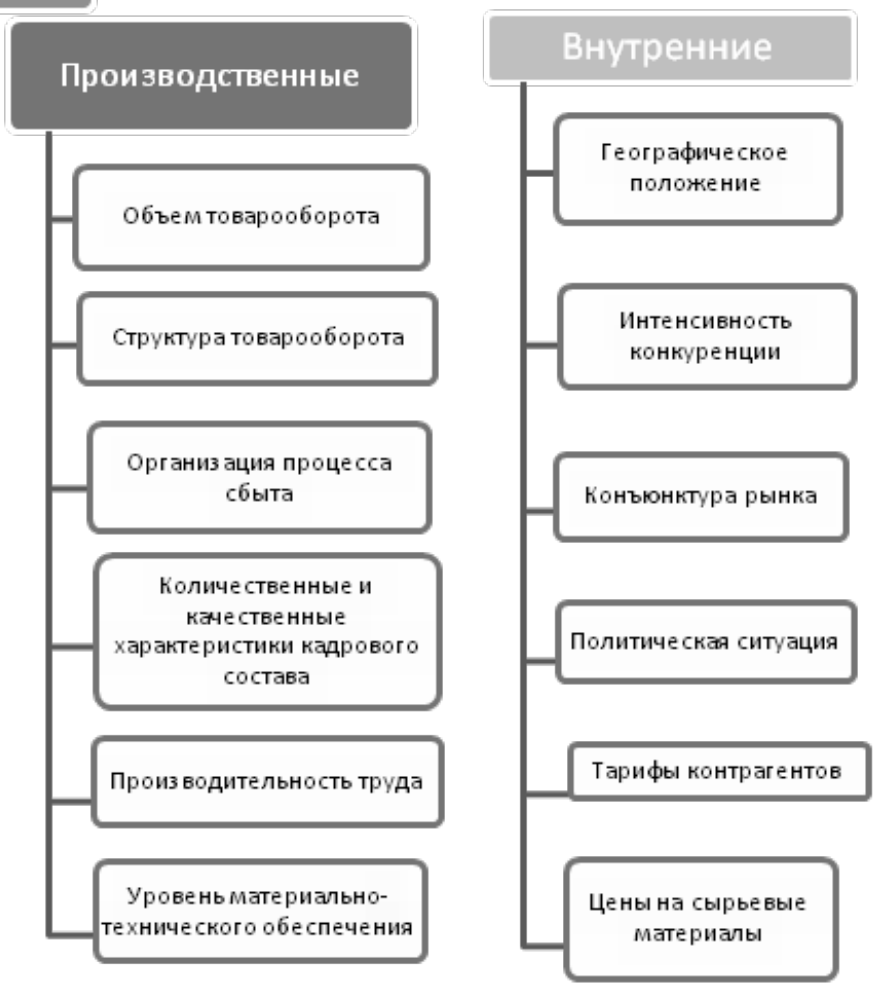

Рис. 1. Факторы воздействия на рентабельность [1] 


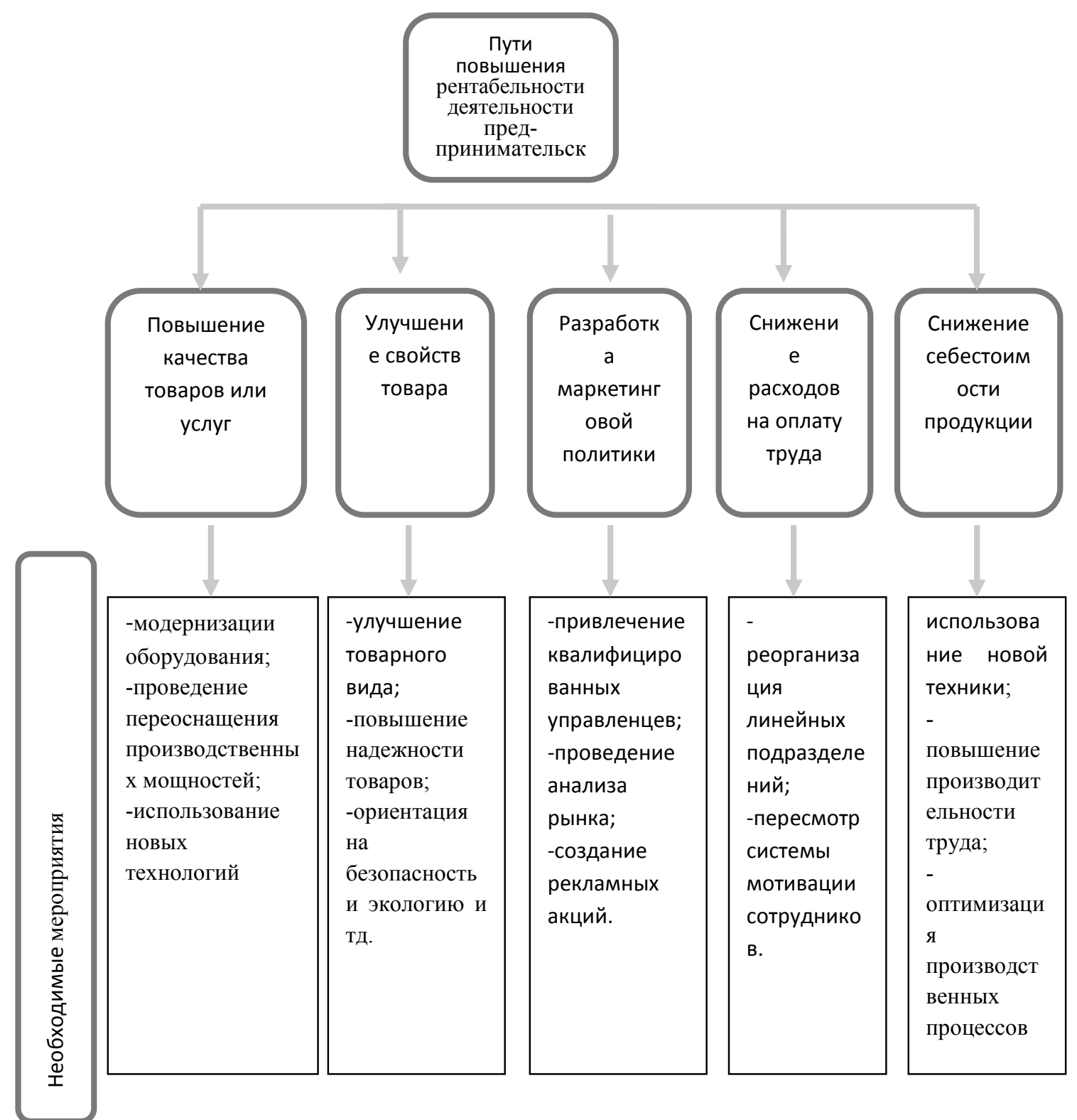

Рис. 2. Пути повышения рентабельности

в аренду неиспользуемый актив. Так получилось с некоторыми крупными заводами в постсоветский период. На территории таких заводов могут располагаться артобъекты, общественные пространства или несколько более мелких предприятий.

Ни одна организация в условиях современной экономики не может осуществлять свою деятельность без основных производственных фондов. Так называют средства производства, которые находятся в распоряжении предприятия. К ним относят здания, сооружения, машины и оборудование, инструменты, производственные и хозяйственные и инвентарь, а также другие разнообразные предметы труда, которые необходимы для деятельности организации. Для осуществление экономически выгодной деятельности предпринимательской структуры все ресурсы, которые необходимы предприятию, должны использоваться максимально эффективно и рационально. Для более подробного изучения вопроса использования основных производственных фондов используется такое показатель, как рентабельность основных фондов.

Рентабельность основных фондов, которая, также, зачастую применяется как рентабельность основных средств рассчитывается как отношение чистой прибыли к стоимости основных средств, умноженное на 100 процентов. Составляющие показатели можно найти в элементах бухгалтерской отчетности. Показатель чистой прибыли зафиксирован в «Отчете о финансовых результатах» исследуемого предприятия, а среднее значение основных производственных фондов находится в форме 
№1 «Бухгалтерский баланс» Этот показатель является интегрированным, или, можно сказать, результатным показателем эффективности производственной деятельности организации.

В экономике всего государства, оптимальное использование основных фондов, играет очень важную роль. Одной из важнейших целей управления эффективностью основных средств является важность обеспечения организационных и экономических условий для эффективности применения конкретных предметов труда. Также расчет рентабельности основных фондов позволяет провести анализ доходности от использования основных производственных фондов, которые в реальном выражении непосредственно принимают участие в производстве товаров или услуг.

Рентабельность основных производственных фондов, как и многие другие показатели рентабельности, способен изменяться под воздействием различных факторов, среди них фондоотдача, скорость оборота основных фондов и т. д. Так, например, если фондоотдача основных средств высокая, то исследуемый показатель будет выше.

Если говорить о нормативных значениях показателя рентабельности основных средств, стоит отметить, что если он принимает отрицательное значение, то деятельность данной организации в обращении с основными фондами не является эффективной. Соответственно, чем выше показатель рентабельности основных производственных фондов, тем эффективней деятельность организации. Однако, не всегда стабильно высокая рентабельность основных средств свидетельствует об эффективности деятельности, например, такое явление может говорить о завышенных ценах на продукцию, расположение организации в низкоконкурентном рыночном сегменте или недостаточное использование инвестиционного потенциала.

Повышение эффективности работы компании должно стать приоритетной задачей при планировании и реализации управленческих решений. В таком случае организация сможет повысить свою конкурентоспособность на рынке и улучшить существующую структуру управления и производства продукции.

\section{ЛИТЕРАТУРА}

1. Губенко А.В., Ксенофонтова Т.Ю. Стратегия повышения роли государства в управлении бизнес-процессами на рынке аэропортовых услуг / Journal of Internet Banking and Commerce. 2015. T. 20. № S1. C. 005.

2. KsenofontovaT.Y., KochelevaT.N., BezdudnayaA.G. The interconnection of demographic factors and indicators of the population density in the region territory at the meso-and micro-levels / International Journal of Applied Business and Economic Research. 2016. T. 14. № 10. C. 7301-7313.

3. К Кенофонтова Т.Ю. Методологические основы управления интеллектуальным капиталом как фактором конкурентоспособности экономической системы / монография / Ксенофонтова Т.Ю. ; М-во образования и науки Российской Федерации, Федеральное гос. бюджетное образовательное учреждение высш. проф. образования «Санкт-Петербургский гос. инженерно-экономический ун-т». Красноярск, 2012. 